\title{
Impacto dos fatores de risco para doenças crônicas não transmissíveis na qualidade de vida
}

\author{
The impact of risk factors of non-communicable chronic diseases \\ on quality of life
}

\author{
Maryane Oliveira-Campos ${ }^{1}$ \\ João Felício Rodrigues-Neto ${ }^{2}$ \\ Marise Fagundes Silveira ${ }^{2}$ \\ Daniele Malard Rocha Neves ${ }^{2}$ \\ Janine Moraes Vilhena ${ }^{2}$ \\ Juliana Fonseca Oliveira ${ }^{2}$ \\ Júlio César Magalhães ${ }^{2}$ \\ Daniel Drumond ${ }^{2}$
}

\footnotetext{
${ }^{1}$ Faculdade de Medicina, Universidade Federal de Minas Gerais. Av. Prof. Alfredo Balena 190, Santa Efigênia. 30130-100 Belo Horizonte MG. oliveiracampos.maryane @yahoo.com.br

${ }^{2}$ Universidade Estadual de Montes Claros.
}

\begin{abstract}
Quality of life has become an important yardstick of the impact on health. Its evaluation related to diseases and associated risk factors has increased in the population in general. This article seeks to assess the quality of life and the presence of the association with risk factors for non-communicable chronic diseases in the urban population of the city of Montes Claros in the State of Minas Gerais. It is a population-based cross-sectional study. The sample was calculated considering the prevalence of risk factors of overweight of $30 \%$ and corrected for a variation of $30 \%$. Cluster sampling was conducted on urban census sectors. Seventeen census sectors were selected and 648 residents aged e" 18 years were interviewed. The risk factors for non-communicable chronic diseases as well as the quality of life were verified using the SF-36 questionnaire. Some risk factors were associated with the lowest scores on the SF-36 questionnaire. The presence of some risk factors for chronic diseases has an impact on quality of life.
\end{abstract}

Key words Quality of life, SF-36, Risk factors, Chronic disease
Resumo A qualidade de vida tornou-se uma importante medida de impacto em saúde. Sua avaliação relacionada às doenças e seus fatores de risco tem acrescido na população em geral. Objetivo: Verificar a qualidade de vida e a presença de associação com fatores de risco para as doenças crônicas não transmissiveis na população urbana do município de Montes Claros, Minas Gerais. Métodos: Estudo transversal de base populacional. A amostra foi calculada considerando-se a prevalência do fator de risco sobrepeso de $30 \%$ e corrigida para um efeito de delineamento de 30\%. Amostragem por conglomerados de setores censitários urbanos. Sorteou-se 17 setores censitários e entrevistou-se 648 moradores de idade maior ou igual a 18 anos. Os fatores de risco para as doenças crônicas não transmissiveis foram verificadas e a qualidade de vida também através do SF-36. Resultados: Alguns dos fatores de risco foram associados a piores escores no questionário SF-36. Conclusão: a presença de alguns fatores de risco para as doenças crônicas tem impacto na qualidade de vida.

Palavras-chave Qualidade de vida, SF-36, Fatores de risco, Doenças crônicas 


\section{Introdução}

As repercussões do desenvolvimento científico e tecnológico nas condições de vida da população têm levado ao aumento da expectativa de vida, expondo a população a um maior risco de desenvolver doenças crônicas não transmissíveis (DCNT). Tanto em países desenvolvidos quanto naqueles em desenvolvimento, estas doenças ocupam as primeiras posições nas estatísticas de mortalidade mundiais ${ }^{1,2}$. Projeções para o ano de 2020 revelam que a mortalidade por doenças crônico-degenerativas representará $73 \%$ dos óbitos no mundo ${ }^{3}$.

O Brasil experimentou acentuadas transformações no contexto do século XX. Por influência do crescimento do comércio internacional instalaram-se formas capitalistas de produção, trabalho e consumo. Diante disso, o processo de industrialização e urbanização desenvolveu-se de forma mais acelerada. Essa dinâmica teve impacto na história social, econômica e cultural, na constituição das diversas regiões brasileiras, produzindo um cenário de desigualdades que perpassa séculos e persiste até a atualidade ${ }^{4}$.

Acompanhando esse cenário, ocorrem intensas e contrastantes modificações na evolução das condições de saúde da população, do conjunto de fatores demográficos, econômicos, sociais e de mudanças nutricionais que interferem no padrão de morbimortalidade no país 5 . O aumento da longevidade populacional chama particular atenção por sua ocorrência de modo acelerado, aumentando também a probabilidade de expressão das enfermidades crônicas, que geralmente se manifestam em idades mais avançadas ${ }^{6}$.

Nos últimos anos, houve um aumento do interesse pela monitorização do nível de qualidade de vida, pela sua importância como indicador prognóstico de morbidade e mortalidade ${ }^{7}$. A QV é reconhecida como um importante indicador de saúde pública, sendo cada vez mais utilizada para controlar o peso da doença em uma população $0^{8-10}$. Entretanto, estudos estabelecem correlação entre baixos níveis de qualidade de vida, doenças crônicas e fatores de risco ${ }^{11,12}$.

Proporcionar saúde significa, além de evitar doenças e prolongar a vida, assegurar meios e situações que ampliem a QV, ou seja, aumentem a capacidade de autonomia e o padrão de bemestar que, por sua vez, são valores socialmente definidos. Nessa perspectiva, a intervenção sanitária refere-se não apenas à dimensão objetiva dos agravos e dos fatores de risco, mas também aos aspectos subjetivos ${ }^{13,14}$.
Este estudo foi proposto na cidade de Montes Claros, localizada ao norte do Estado de Minas Gerais. O município tem uma população de 306.730 habitantes, sendo a maioria, 289.006 habitantes, residentes na região urbana e apresenta um IDH - Índice de Desenvolvimento Humano - igual a $0,783^{15,16}$. O presente estudo tem como objetivo verificar a existência de associação entre a qualidade de vida e os fatores de risco para as doenças crônicas não transmissíveis na população da região urbana de Montes Claros, Minas Gerais, Brasil.

\section{Metodologia}

Este estudo é transversal de base populacional. Foram selecionados indivíduos com idade igual ou superior a dezoito anos de idade, de ambos os sexos e residentes na região urbana de Montes Claros no período de fevereiro a agosto de 2008. Foram impossibilitados de participar do estudo aqueles que apresentaram limitações físicas e/ou mentais -, além das grávidas e dos acamados.

Para determinação do número total de indivíduos da amostragem, considerou-se: (i) o número total de pessoas com idade maior ou igual a 18 anos, residentes na zona urbana de Montes Claros, e que equivale a $224.451^{15}$; (ii) uma prevalência estimada do fator de risco excesso de peso de $30 \%$; (iii) um intervalo de confiança de $95 \%$; (iv) um erro fixado de $5 \%$; e, (v) deff (efeito de desenho) de 1,3. Os cálculos evidenciaram um tamanho mínimo amostral (n) de 648 participantes.

A amostragem foi probabilística por conglomerado em três estágios. O município de Montes Claros apresenta duzentos e dezesseis (216) setores censitários, sendo que os setores não urbanos (rurais) e urbanos especiais (asilos, creches, orfanatos, hospitais, conventos) - que totalizam trinta e cinco (35) instituições - foram excluídos do estudo, restando o equivalente a cento e oitenta e um (181) setores representativos dos domicílios urbanos do município. Destes, 163 são "urbanos não especiais" ( $96 \%$ da população urbana) e 18 "urbanos subnormais" (aglomerados, favelas) representando $4 \% \mathrm{da}$ população urbana.

No primeiro estágio, para a representatividade dos setores censitários urbanos, dentre os 181 setores, foram selecionados por amostragem aleatória 17. Destes, foram sorteados 15 "urbanos não especiais" e 2 "urbanos subnormais".

No segundo estágio, foi sorteada uma quadra dentro de cada setor, iniciando a coleta na 
primeira rua do lado direito. Os domicílios residenciais foram amostrados em ordem de três, sendo o primeiro de cada três visitado. Quando não foi possível realizar a entrevista, o domicílio seguinte era visitado e a contagem reiniciada a partir deste ponto. Após o termino da rua, os entrevistadores viravam à direita seguindo o mapa de cada setor censitário. Em cada domicílio foi entrevistado um morador, sendo a escolha do entrevistado realizada por sorteio aleatório simples, constituindo o terceiro estágio do processo de seleção da amostra. Para compensar as probabilidades desiguais de seleção das unidades em cada um dos três estágios atribuíram-se ponderações diferenciadas aos elementos da amostra, correspondentes ao inverso do produto das probabilidades de inclusão nos diversos estágios de seleção.

\section{Coleta de dados}

Com base na resolução 196/96 do Conselho Nacional de Saúde do Brasil ${ }^{17}$, este estudo passou pela apreciação do Comitê de Ética em pesquisa da Universidade Estadual de Montes Claros, sendo aprovado. Os voluntários receberam informações sobre a metodologia e apresentaram após a leitura e entendimento, o consentimento livre e esclarecido.

O instrumento de coleta de dados foi um questionário semiestruturado contendo investigações sobre os aspectos socioeconômicos e demográficos, fatores de risco para as doenças crônicas não transmissíveis, comorbidades autorreferidas e qualidade de vida.

Foram considerados fatores de risco a atividade física insuficiente, o tabagismo, o consumo excessivo de álcool, o consumo inadequado de frutas e hortaliças, o sobrepeso e a obesidade, níveis alterados de pressão arterial e hiperglicemia ${ }^{18}$.

No nível de atividade física insuficiente foram incluídos indivíduos irregularmente ativos e sedentários, avaliado pelo Questionário Internacional de Atividade Física versão 8 (IPAQ-8) ${ }^{19}$. No nível de tabagismo foram incluídos fumantes e ex-fumantes que abandonaram a prática num período inferior a cinco anos ${ }^{20}$. Como consumo excessivo de álcool considerou-se a ingestão diária média de bebida maior ou igual a duas doses padronizadas para homens e maior ou igual a uma dose padronizada para mulheres. Como uma dose padronizada considerou-se a ingestão de meia garrafa ou uma lata de cerveja, um cálice de vinho ou uma dose de bebida desti$\operatorname{lada}^{21}$. O baixo consumo de frutas, verduras e legumes foi definido como a ingestão destes com uma frequência menor a de cinco vezes por semana, avaliado através do questionário de frequência alimentar ${ }^{22}$. O índice de massa corporal (IMC) entre 25 e $29,9 \mathrm{~kg} / \mathrm{m}^{2}$ foi classificado como sobrepeso e o IMC maior ou igual a $30 \mathrm{~kg} / \mathrm{m}^{2}$, classificado como obesidade. O cálculo do IMC foi realizado a partir de dados obtidos com uma balança digital G-Tech e um estadiômetro portátil do tipo trena, com o indivíduo livre de sapatos e roupas pesadas ${ }^{23}$. Os níveis alterados de pressão arterial foram aqueles que consideraram a pressão arterial sistólica maior ou igual a 140 $\mathrm{mmHg}$ e/ou pressão arterial diastólica maior ou igual a $90 \mathrm{mmHg}$, independentemente do uso de anti-hipertensivos ${ }^{24}$. A medida de pressão arterial foi realizada através de um aparelho de pressão digital modelo MF-81, da marca Mark of Fitness, por duas vezes, e a média das duas foi utilizada na análise. Hiperglicemia foi considerada a glicemia casual maior que $200 \mathrm{mg} / \mathrm{dL}$, obtido através de um glicosímetro da marca Prestige ${ }^{25,26}$.

Para a avaliação da Qualidade de Vida foi utilizado o questionário The Medical Outcomes Study 36-Item Short Form- Health Survey (SF-36), traduzido e validado no Brasil ${ }^{27}$. O SF-36 é um questionário multidimensional que engloba 8 domínios, formado por 36 itens. 1. Capacidade Funcional (CF), que investiga a presença e a extensão das dificuldades relacionadas a atividades diárias, como subir escadas, tomar banho, etc., com dez itens; 2. Limitações por Aspectos Físicos (AF), que pesquisa limitações e o quanto elas dificultam as tarefas diárias ou o trabalho, com quatro itens; 3. Dor (DR), que investiga a presença de sofrimento, fundamentando-se na intensidade da dor, extensão ou interferência dela nas atividades diárias, com dois itens; 4. Estado Geral de Saúde (EGS), que são aspectos gerais relacionados à percepção de saúde, com cinco itens; 5 . Vitalidade (VT), que considera o nível de vigor, a energia e o cansaço, com quatro itens; 6 . Aspectos Sociais (AS), que demonstram o quanto um problema físico ou emocional interfere nas atividades sociais, com dois itens; 7. Aspectos Emocionais (AE), que investigam a participação em atividades de trabalho e tempo dispensado em decorrência de problemas emocionais, com três itens; 8. Saúde Mental (SM), que investiga a presença de ansiedade, depressão, alterações do comportamento, descontrole emocional e bem-estar psicológico, com cinco itens; Além destes componentes, o questionário engloba mais uma questão de avaliação comparativa entre as condições de saúde atual e de um ano atrás, que não entra na pon- 
tuação de nenhuma das oito dimensões explicitadas. Para a avaliação de seus resultados, após sua aplicação, foi atribuído um valor a cada questão, o qual, posteriormente, foi transformado num escore de 0 a 100, onde 0 corresponde a um pior estado de saúde, e 100 a um melhor, sendo analisada cada dimensão em separado.

\section{Análise estatística}

Neste estudo buscou-se analisar, através de modelos de regressão linear múltipla, a associação entre qualidade de vida e os fatores de risco para as doenças crônicas não transmissíveis.

As medidas de QV (escores das dimensões do SF-36) variam de 0 a 100 e foram consideradas variáveis dependentes. As características socioeconômicas, demográficas e os fatores de risco foram considerados variáveis independentes.

As variáveis foram descritas através de medidas de tendência central e dispersão. Realizou-se análise bivariada por meio de testes não paramétricos Mann-Witney e Kuskall-Wallis para verificar associação entre as variáveis independentes e dependentes. As variáveis que apresentaram valor-p menor que 0,20 foram selecionadas para o modelo multivariado, no qual se procedeu à análise de regressão linear múltipla. Em todas as etapas foi adotada a correção pelo efeito do desenho utilizando-se o módulo complex samples do programa estatístico Statistical Package for the Social Sciences (SPSS), versão 15.0.

\section{Resultados}

Foram entrevistados 648 indivíduos de ambos os sexos, sendo $478(73,8 \%)$ do sexo feminino. A média de idade foi de 43 anos, sendo a idade mínima de 18 e a máxima de 97 . O tempo médio para completar a entrevista foi de 17 minutos.

Entre as características socioeconômicas e demográficas da amostra, a faixa etária entre 1844 e 45-64 anos apresentou 592 indivíduos $(91,3 \%)$. Declararam a cor da pele como não branca um total de 437 indivíduos (67,4\%). Em situação conjugal casado ou união consensual, 366 indivíduos (56,5\%). Em atividade profissional, 437 indivíduos $(67,4 \%)$. Apresentavam até 11 anos de escolaridade 555 indivíduos (85,6\%) e relataram renda familiar mensal de até 3 salários mínimos uma soma de 405 indivíduos (62,5\%); conforme descrito na Tabela 1.

A prevalência dos fatores de risco na população estudada apontou um baixo consumo de frutas (77,3\%) [IC95\% 74,0-80,0]; um baixo consumo de verduras e legumes (55,1\%) [IC95\% 51,2-58,9]; tabagismo (16,2\%) [IC95\% 13,319,0]; sobrepeso (32,6\%) [IC95\% 29,0-36,2]; obesidade (18,7\%) [IC95\% 15,7-21,7]; nível insuficiente de atividade física (26,7\%) [IC95\% 23,330,1]; hiperglicemia (11,6\%) [IC95\% 9,1-14,7]; níveis pressóricos elevados (32,5\%) [IC95\% 28,9$36,1]$; e consumo excessivo de álcool $(5,7 \%)$ [IC95\% 3,9-7,4].

A média dos escores das dimensões do questionário SF-36 na população adulta da região urbana de Montes Claros está demonstrada na Tabela 2. Os escores de maior comprometimento foram observados nos domínios VT $(65,7 \pm$ $22,5)$, DR $(67,6 \pm 21,4)$, EGS $(65,8 \pm 28,4)$ e SM $(67,6 \pm 21,4)$.

O modelo da Regressão Linear Múltipla está apresentado na Tabela 3. Entre as características socioeconômicas e demográficas, o sexo feminino apareceu associado a piores escores de QV em quase todas as dimensões, em relação ao masculino, com exceção ao domínio EGS. Em relação à faixa etária de 18-44 anos, os indivídu-

Tabela 1. Perfil socioeconômico e demográfico da população adulta na região urbana de Montes Claros, Minas Gerais, $(\mathrm{n}=648), 2008$.

\begin{tabular}{lrr}
\hline \multicolumn{1}{c}{ Variáveis } & $\mathbf{n}$ & $\%$ \\
\hline Sexo & & \\
$\quad$ Feminino & 478 & 73,8 \\
$\quad$ Masculino & 170 & 26,2 \\
Faixa etária & & \\
$\quad 18$ - 44 anos & 330 & 50,9 \\
$\quad 45$ - 64 anos & 262 & 40,4 \\
$\quad$ 65 anos & 56 & 8,7 \\
Cor da pele autodeclarada & & \\
$\quad$ Branca & 143 & 22,1 \\
$\quad$ Não branca & 437 & 67,4 \\
$\quad$ Não informaram & 68 & 10,5 \\
Situação conjugal & & \\
$\quad$ Casado ou união consensual & 366 & 56,7 \\
$\quad$ Outros & 280 & 43,3 \\
Em atividade profissional & & \\
$\quad$ Sim & 437 & 67,4 \\
$\quad$ Não & 211 & 32,6 \\
Anos de escolaridade & & \\
$\quad<11$ anos & 555 & 85,6 \\
$\quad \geq 12$ anos & 93 & 14,4 \\
Renda familiar mensal & & \\
$\quad<3$ salários mínimos & & \\
$\quad \geq 3$ e 6 salários mínimos & 905 & 62,5 \\
$\quad$ > salários mínimos & 98 & 15,1 \\
$\quad$ Não informaram & 61 & 9,4 \\
\hline
\end{tabular}


Tabela 2. Escores das dimensões do SF-36 na população adulta da região urbana de Montes Claros, Minas Gerais, Brasil, ( $\mathrm{n}=648), 2008$.

\begin{tabular}{|c|c|}
\hline Dimensões do SF-36 & $\operatorname{Média}^{*} \pm \mathrm{DP}^{*}$ \\
\hline $\mathrm{CF}$ & $\pm 24,3$ \\
\hline $\mathrm{AF}$ & $75,3 \pm 39,8$ \\
\hline $\mathrm{DR}$ & $65,8 \pm 28,4$ \\
\hline EGS & $67,6 \pm 21,4$ \\
\hline VIT & $65,7 \pm 22,5$ \\
\hline AS & $73,8 \pm 22,8$ \\
\hline $\mathrm{AE}$ & $72,7 \pm 41,2$ \\
\hline SM & $67,0 \pm 21,6$ \\
\hline
\end{tabular}

"correção pelo efeito de desenho (deff)

$\mathrm{DP}=$ desvio padrão

$\mathrm{CF}=$ capacidade funcional, $\mathrm{AF}=$ limitações por aspectos

físicos, $\mathrm{DR}=$ dor, $\mathrm{EGS}=$ estado geral de saúde, $\mathrm{VT}=$ vitalidade, $\mathrm{AS}=$ aspectos sociais, $\mathrm{AE}=$ aspectos emocionais, $\mathrm{SM}=$ saúde mental.

os de 45-64 anos apresentaram piores escores na CF e DR. Os indivíduos com idade maior ou igual a 65 anos também permaneceram relacionados a piores escores de QV dos domínios CF e VT.

Em relação aos indivíduos com 12 anos ou mais de escolaridade, aqueles que relataram níveis inferiores de estudo obtiveram piores escores na dimensão EGS, AE e SM. Em relação à renda familiar mensal, permaneceu associada a uma pior QV os indivíduos que relataram renda familiar mensal menor que 3 salários mínimos na dimensão CF e renda familiar maior que 6 salários mínimos nas dimensões AF e EGS.

Entre as comorbidades autorreferidas, os indivíduos que relatam a presença de hipertensão arterial apresentaram uma pior QV nas dimensões CF, EGS, VT, AS, AE, SM; de doenças reumatológicas nas dimensões CF, DR, EGS, AS, AE; doenças vasculares nas dimensões AF, EGS, VT; de doença cardíaca nas dimensões $\mathrm{CF}, \mathrm{AF}, \mathrm{DR}$, AS; doença renal nas dimensões CF, AF, VT; de doenças psiquiátricas nas dimensões AF, EGS, VT, AS, AE, SM e diabetes mellitus na dimensão EGS.

Entre os fatores de risco, obtiveram os piores escores de QV os tabagistas, os obesos e os indivíduos que apresentaram um consumo excessivo de álcool. Os tabagistas apresentaram piores escores de QV em relação aos não tabagistas, nas dimensões AF, EGS, VT, AS e SM. Os obesos apresentaram pior escore de QV em relação aos eutróficos na dimensão CF. Os indivíduos que apresentaram um consumo excessivo de álcool em relação aos que não consomem ou consomem moderadamente, obtiveram pior QV na dimensão CF.

\section{Discussão}

No Brasil, além do aumento da prevalência por doenças crônicas, há o desafio da coexistência de novos e velhos agravos, uma vez que continua elevada a magnitude das doenças infecto-parasitárias e desigualdades regionais proporcionadas pelas diferenças na distribuição de renda, escolaridade e políticas públicas ${ }^{28}$. Há importantes estudos populacionais brasileiros sobre aspectos socioeconômicos e prevalência dos fatores de risco para a DCNT ${ }^{29-34}$. Entretanto, em estudos nacionais e internacionais, pouco se sabe sobre o impacto desses fatores de risco para as DCNT na QV da população em geral.

Em relação ao sexo, as mulheres deste estudo apresentaram escores mais baixos de QV em quase todos os domínios do SF-36, como verificado em outros estudos ${ }^{35-37}$. Uma alta proporção de angústia, depressão, estresse, ansiedade e distúrbios no sono são encontrados em mulheres em idade reprodutiva. Além das diferenças biológicas, assumem novos papéis na sociedade, têm acúmulo de funções e apresentam uma percepção subjetiva de valor ao estado interior $^{38,39}$. Outro aspecto a ser considerado é o aumento da expectativa de vida das mulheres, que faz com que muitas idosas passem pela experiência de debilitação biológica ${ }^{40}$. À medida que aumenta a idade, a QV é fortemente determinada por sua habilidade de manter autonomia e independência ${ }^{41}$.

Na faixa etária de 45-64 já se evidencia o processo de declínio de QV nas dimensões CF e DR. A Dor pode estar relacionada à carga de trabalho nessa faixa etária, na qual os indivíduos encontram-se economicamente ativos. Outro aspecto a ser evidenciado em relação à faixa etária, em indivíduos com idade maior ou igual a 65 anos, é a existência de um impacto expressivo nas dimensões CF e VT, que pode ser evidenciada pelo declínio das condições funcionais causada pelo envelhecimento ${ }^{42}$. Além disso, muitos idosos necessitam de pessoas em seu cuidado, familiares, parentes, amigos e cuidadores.

A qualidade de vida é influenciada pela escolaridade e pela renda dos indivíduos, como encontrado neste e em outros estudos populacionais realizados em Cingapura e na Alemanha ${ }^{11,12}$. Assim, o bem-estar envolve outros aspectos além de saúde. Dessa forma, a QV está diretamente relacionada com níveis de educação e distribuição de renda ${ }^{43}$.

Em relação aos aspectos socioeconômicos e demográficos, o fato de ser mulher e ter baixa 
Tabela 3. Modelo da Regressão Linear Múltipla, com correção pelo efeito de desenho, para as dimensões de qualidade de vida do SF-36 na população da região urbana de Montes Claros, Minas Gerais, Brasil $(\mathrm{n}=648)$.

\begin{tabular}{|c|c|c|c|c|c|c|c|c|}
\hline & \multicolumn{8}{|c|}{ Dimensões de qualidade de vida do SF-36 } \\
\hline & \multicolumn{2}{|c|}{ CF } & \multicolumn{2}{|c|}{ AF } & \multicolumn{2}{|c|}{ DR } & \multicolumn{2}{|c|}{ EGS } \\
\hline & $\beta$ & valor $\mathrm{p}$ & $\beta$ & valor $\mathrm{p}$ & $\beta$ & valor $\mathrm{p}$ & $\beta$ & valor $\mathrm{p}$ \\
\hline \multicolumn{9}{|l|}{$\begin{array}{l}\text { Características socioeconômicas } \\
\text { e demográficas }\end{array}$} \\
\hline \multicolumn{9}{|l|}{ Sexo } \\
\hline Feminino & $-4,45$ & 0,003 & $-9,44$ & 0,00 & $-5,23$ & 0,03 & - & - \\
\hline \multicolumn{9}{|l|}{ Faixa etária } \\
\hline 45-64 anos & $-8,76$ & 0,00 & - & - & $-5,98$ & 0,00 & - & - \\
\hline$\geq 65$ anos & $-21,0$ & 0,00 & - & - & - & - & - & - \\
\hline Anos de escolaridade & - & - & - & - & - & - & $-6,69$ & 0,00 \\
\hline \multicolumn{9}{|l|}{ Renda familiar mensal } \\
\hline$<3$ salários mínimos & $-8,20$ & 0,00 & - & - & - & - & - & - \\
\hline 3 a 6 salários mínimos & - & - & $-10,6$ & 0,01 & - & - & $-4,81$ & 0,02 \\
\hline \multicolumn{9}{|l|}{ Fatores de risco } \\
\hline Tabagismo & - & - & $-16,6$ & 0,00 & - & - & $-7,18$ & 0,00 \\
\hline Sobrepeso & - & - & - & - & - & - & - & - \\
\hline Obesidade & $-5,99$ & 0,01 & - & - & - & - & - & - \\
\hline Níveis pressóricos alterados & - & - & $-9,02$ & 0,00 & - & - & - & - \\
\hline Nível insuficiente de atividade física & - & - & - & - & - & - & - & - \\
\hline Hiperglicemia & - & - & - & - & - & - & - & - \\
\hline \multirow[t]{4}{*}{ Consumo excessivo de álcool } & $-7,13$ & 0,01 & - & - & - & - & - & - \\
\hline & \multicolumn{8}{|c|}{ Dimensões de qualidade de vida do SF-36 } \\
\hline & \multicolumn{2}{|c|}{ VT } & \multicolumn{2}{|c|}{ AS } & \multicolumn{2}{|c|}{$\mathrm{AE}$} & \multicolumn{2}{|c|}{ SM } \\
\hline & $\beta$ & valor $\mathrm{p}$ & $\beta$ & valor $\mathrm{p}$ & $\beta$ & valor $p$ & $\beta$ & valor $\mathrm{p}$ \\
\hline \multirow{2}{*}{\multicolumn{9}{|c|}{$\begin{array}{l}\text { Características socioeconômicas } \\
\text { e demográficas } \\
\text { Sexo }\end{array}$}} \\
\hline & & & & & & & & \\
\hline Feminino & $-8,87$ & 0,00 & $-6,47$ & 0,00 & $-12,4$ & 0,00 & $-8,00$ & 0,00 \\
\hline \multicolumn{9}{|l|}{ Faixa etária } \\
\hline 45-64 anos & - & - & - & - & - & - & - & - \\
\hline$\geq 65$ anos & $-6,92$ & 0,04 & - & - & - & - & - & - \\
\hline Anos de escolaridade & - & - & - & - & $-8,06$ & 0,03 & $-5,95$ & 0,00 \\
\hline \multicolumn{9}{|l|}{ Renda familiar mensal } \\
\hline < 3 salários mínimos & - & - & - & - & - & - & - & - \\
\hline 3 a 6 salários mínimos & - & - & - & - & - & - & - & - \\
\hline \multicolumn{9}{|l|}{ Fatores de risco } \\
\hline Tabagismo & $-6,94$ & 0,01 & $-6,80$ & 0,00 & - & - & $-6,61$ & 0,00 \\
\hline Sobrepeso & - & - & - & - & - & - & - & - \\
\hline Obesidade & - & - & - & - & - & - & - & - \\
\hline Níveis pressóricos alterados & - & - & - & - & - & - & - & - \\
\hline Nível insuficiente de atividade física & - & - & - & - & - & - & - & - \\
\hline Hiperglicemia & - & - & - & - & - & - & - & - \\
\hline Consumo excessivo de álcool & - & - & - & - & - & - & - & - \\
\hline
\end{tabular}

$\beta=$ coeficiente de regressão. $\mathrm{CF}=$ capacidade funcional, $\mathrm{AF}=$ limitações por aspectos físicos, $\mathrm{DR}=$ dor, $\mathrm{EGS}=$ estado geral de saúde, $\mathrm{VT}=$ vitalidade, $\mathrm{AS}=$ aspectos sociais, $\mathrm{AE}=$ aspectos emocionais, $\mathrm{SM}=$ saúde mental.

Nota: ajustado pelas comorbidades auto-referidas (hipertensão arterial, dislipidemia, doença reumatológica, vascular, cardíaca, renal, psiquiátrica, pulmonar, diabetes mellitus e neoplasia).

escolaridade comprometeu tanto a saúde física a quanto mental. Apresenta comprometimento da QV somente na saúde física os indivíduos com idade avançada e renda familiar menor que 3 salários mínimos ou maior que 6 salários mínimos. A QV pode ser influenciada por infraestrutura, logística e serviços públicos. Ações como investimento em educação, geração de empre- 
gos, acesso a serviços de saúde e programas direcionados para indivíduos com idade avançada são ações diretas que podem proporcionar uma melhor QV. Um aspecto relevante a ser evidenciado é que a percepção de QV pode ser influenciada por fatores culturais, socioeconômicos, expectativa e realidade da vida ${ }^{44-48}$.

Este estudo verificou que fatores de risco, níveis pressóricos alterados, obesidade e consumo excessivo de álcool estão associados a uma pior QV. Em indivíduos com níveis pressóricos alterados, o domínio AF foi acometido. Esta associação ocorre independentemente do indivíduo ter conhecimento ou não sobre a sua hipertensão arterial. A QV pode ser acometida antes de o indivíduo ter o diagnóstico de hipertensão arterial. Estudos realizados nos Estados Unidos da América apontam que o risco relativo de uma pessoa ter a comorbidade é bem maior se ela apresentar baixa capacidade física ${ }^{49,50}$. A hipertensão talvez possa resultar em uma limitação nas atividades diárias individuais. Embora a hipertensão arterial seja caracterizada como uma doença assintomática, indivíduos com níveis pressóricos alterados têm maior frequência de sintomas de cefaleia, ansiedade, astenia, distúrbios do sono, que os normotensos ${ }^{51}$. Estudos mostram uma associação entre um bom controle da HAS com tratamento e uma melhora da $\mathrm{QV}^{52,53}$. O controle dos níveis pressóricos e a educação em saúde têm sido apontados como uma das formas para estimular a adesão ao tratamento e melhora da QV.

Além dos níveis pressóricos alterados, a obesidade é outro fator de risco que está associado ao domínio CF. As condições debilitantes da obesidade, tais como osteoartrite, dificuldades respiratórias, problemas músculo-esqueléticos e infertilidade podem alterar a percepção subjetiva do paciente sobre sua saúde física ${ }^{54,55}$.

A QV diminui proporcionalmente ao aumento do IMC e as percepções de saúde podem afetar os esforços dos indivíduos para manter ou alterar seu peso $^{56}$. Apesar de o presente estudo apresentar comprometimento relacionado somente à saúde física, em revisão bibliográfica, na maioria das pesquisas os obesos apresentam, de forma concomitante, comprometimento na saúde mental ${ }^{57}$.

Neste estudo, encontramos um número expressivo de pessoas com excesso de peso $(51,7 \%)$, o que pode estar associado ao padrão alimentar. $\mathrm{O}$ consumo de alimentos industrializados na população adulta brasileira teve um aumento expressivo em 2003 , atingindo $40,6 \%{ }^{58}$. No presente estudo, encontramos na população de
Montes Claros alto percentual de indivíduos com baixo consumo de frutas, verduras $(77,3 \%)$ e legumes $(55,1 \%)$.

O tabagismo apresentou-se como o fator de risco de maior impacto na $\mathrm{QV}$, acometendo cinco das oito dimensões levadas em conta neste estudo. Embora não tenhamos classificado a gravidade da dependência do tabaco em nossos entrevistados, outros estudos mostram que a QV é comprometida, tanto em tabagistas leves ${ }^{59}$, quanto nos crônicos ${ }^{60}$.

O presente estudo encontrou associações tanto nos domínios relacionados à saúde física quanto mental, correlação também evidenciada em outros $^{61,62}$. Porém, neste houve um maior comprometimento da saúde mental. Indivíduos com má saúde mental têm maior probabilidade de fumar ${ }^{63}$ e apresentam alta frequência de comorbidades psiquiátricas ${ }^{64,65}$, sendo importante ressaltar que quando há uma cessação do hábito de fumar há uma melhora da $\mathrm{QV}^{66,67}$.

De acordo com a Organização Mundial de Saúde $(\mathrm{OMS})^{68}$, a mortalidade e a limitação da condição funcional, associada ao consumo de bebidas alcoólicas, superam aquelas associadas ao tabagismo. Embora os tabagistas tenham apresentado comprometimento de um número expressivo de domínios, neste estudo não encontramos associação no de Capacidade Funcional. Indivíduos que realizavam o consumo excessivo de álcool tiveram um escore inferior na QV neste domínio, como também observado pela OMS.

Em outros estudos populacionais, o consumo excessivo de álcool foi associado a outros domínios do SF-36 $6^{68,69}$. A intervenção terapêutica está relacionada a uma melhoria da QV e apresenta-se como uma oportunidade de intervir antes que o padrão de uso de álcool provoque danos à saúde do paciente ${ }^{70,71}$.

A medida de QV tem sido utilizada na tomada de decisão em relação à intervenção, concepção, gestão e alocação de recursos em saúde ${ }^{43,44}$. Diante disso, é possível melhorá-la com medidas de intervenções populacionais e individuais. Observa-se que na saúde física, nosso estudo encontrou pior QV nos indivíduos que apresentaram níveis pressóricos alterados, obesidade e consumo excessivo de álcool. Contudo, o fato de ser tabagista acometeu tanto a saúde física quanto mental.

Ressaltamos a importância do planejamento de políticas públicas direcionadas à população em ações coletivas, como melhor acesso aos serviços de saúde, programas de educação em saúde, campanhas que atuem no aspecto cognitivo, refletin- 
do em modificação ou adoção de hábitos favoráveis a um estilo de vida mais saudável, entre outras. Além disso, proporcionar estímulos para ações individuais, como o controle dos níveis pressóricos, orientações para aspectos nutricionais visando à redução do peso, mudança de hábitos de tabagistas e de consumidores de álcool.

Uma limitação deste estudo é o fato das entrevistas terem sido realizadas exclusivamente no período diurno, em que grande parte do público masculino poderia estar ausente de suas residências, exercendo suas funções no trabalho, o que pode ter contribuído para o percentual superior de mulheres. Outra observação relevante, que poderia consistir numa outra limitação do estudo, é a ausência de medida da QV na população geral brasileira para a padronização de QV encontrada em Montes Claros.

\section{Colaboradores}

M Oliveira-Campos e JF Rodrigues-Neto participaram de todas as etapas no estudo. J Vilhena, D Malard, J Oliveira, J Magalhães e D Drummond, na coleta de dados, metodologia e resultados. M Fagundes, na análise estatística, metodologia e resultados.

\section{Agradecimentos}

À FAPEMIG pelo financiamento e concessão de bolsa de pesquisa de mestrado à Oliveira-Campos e a CAPES de doutorado.

\section{Conclusão}

Este estudo verificou que a presença de alguns fatores de risco para as DCNT tem associação com pior QV. Os níveis pressóricos alterados, a obesidade e o consumo excessivo de álcool estão associados a uma pior saúde física. O tabagismo está associado a uma pior saúde física e mental.

Os resultados deste estudo podem subsidiar o desencadeamento de ações coletivas e individuais em indivíduos rastreados com fatores de risco para as doenças crônicas. Assim, esperamos contribuir para estimular ações de promoção de saúde, em um foco de não apenas diminuir o risco de DCNT, mas aumentar as chances de melhoria da QV da população.

\section{Referências}

1. Lopez AD. The evolution of the Global Burden of Disease framework for disease, injury and risk factor quantification: developing the evidence base for national, regional and global public health action. Globalization and Health 2005; 1(5):1-8.

2. Wilson KM, Satterfield DW. Where Are We to Be in These Times? The Place of Chronic Disease Prevention in Community Health Promotion. Prev Chronic Dis 2007; 4(3):A74.

3. Mathers CD, Loncar D. Projections of Global Mortality and Burden of Disease from 2002 to 2030. PLoS Med 2006; 3(11):442.

4. Santos M. A urbanização brasileira. 2a Edição. São Paulo: Hucitec; 1994.

5. Prata PR. The Epidemiologic Transition in Brazil. Cad Saude Publica 1992; 8(2):168-175.

6. Achutti A, Azambuja MIR. Doenças crônicas nãotransmissíveis no Brasil: repercussões do modelo de atenção à saúde sobre a seguridade social. Cien Saude Colet 2004; 9(4):833-840.

7. Peters R. Quality-of-life measures as predictors of mortality and morbidity. Curr Hypertens Rep 2001; 3(6):458-461.

8. Brown DW, Balluz LS, Heath GW, Moriarty DG, Ford ES, Giles WH, Mokdad AH. Associations between recommended levels of physical activity and health-related quality of life. Findings from the 2001 Behavioral Risk Factor Surveillance System (BRFSS) survey. Prev Med 2003; 37(5):520-528.

9. Brown DW, Balluz LS, Giles WH, Beckles GL, Moriarty DG, Ford ES, Mokdad AH: Diabetes mellitus and health-related quality of life among older adults. Findings from the behavioral risk factor surveillance system (BRFSS). Diabetes Res Clin Pract 2004; 65(2):105-115. 
10. Moriarty DG, Zack MM, Kobau R. The Centers for Disease Control and Prevention's Healthy Days Measures - Population tracking of perceived physical and mental health over time. Health Qual Life Outcomes 2003; 1(1):37.

11. Jiang Y, Hesser JE. Patterns of health-related quality of life and patterns associated with health risks among Rhode Island adults. Health Qual Life Outcomes 2008; 6:49.

12. Wang H, Beyer M, Gensichen J, Gerlach FM. Health-related quality of life among general pratice patients with differing chronic diseases in Germany: Cross sectional survey. BMC Public Health 2008; $8: 246$.

13. Buss PM. Promoção da saúde e qualidade de vida. Cien Saude Colet 2000; 5(1):163-177.

14. Seidl EMF, Zannon CMLC. Qualidade de vida e saúde: aspectos conceituais e metodológicos. Cad Saude Publica 2004; 20(2):580-588.

15. Instituto Brasileiro de Geografia e Estatística (IBGE). Censo Demográfico 2000. [acessado 2007 ago 30]. Disponível em: http://www.ibge.gov.br

16. Fundação João Pinheiro (FJP). Atlas do desenvolvimento humano no Brasil. Software; 2003.

17. Brasil. Ministério da Saúde (MS). Conselho Nacional de Saúde. Resolução no 196 de 10 de outubro de 1996. Diretrizes e Normas Regulamentadoras de Pesquisas Envolvendo Seres Humanos. Diário Oficial da União 1996; out 16.

18. World Health Organization (WHO). The World Health Report, 2002: reducing risks, promoting healthy life. NLM Classification. WA 540.1. Geneva: WHO; 2002.

19. Matsudo S, Araújo T, Andrade D, Andrade E, Oliveira LC, Braggion G. Questionário internacional de atividade física (IPAQ): estudo de validade e reprodutibilidade no Brasil. Rev Bras de Atividade Física e Saúde 2001; 6(2):5-18

20. World Health Organization (WHO). International guide for monitoring alcohol consumption and related harm. Geneva: WHO; 2000.

21. Furlan-Viebig R, Pastor-Valero M. Desenvolvimento de um questionário de freqüência alimentar para o estudo de dieta e doenças não transmissíveis. Rev Saude Publica 2004; 38(4):581-584.

22. World Health Organization (WHO). Food and Agricultural Organization (FAO). Expert Report on Diet, Nutrition and the Prevention of Chronic Diseases. Geneva: WHO, FAO; 2003. (WHO Technical Report Series, n. 916).

23. World Health Organization (WHO). Obesity: preventing and managing the global epidemic: report of a WHO consultation on obesity. Geneva: WHO 1998. (WHO/NUT/NCD/98.1).

24. Joint National Committee on Prevention, Detection, evaluation and treatment of high blood pressure. The Seventh Report. JAMA 2003; 289(19):2560-2572.

25. American Diabetes Association. Diagnosis and Classification of Diabetes Mellitus. Diabetes Care 2008; 31(1):55-60

26. McGregor MS, Pinkham C, Ahroni J, Herter CD, Doctor JD. The American Diabetes Association Risk Tests for diabetes. Diabetes Care 1995; 18(4):585-586.

27. Ciconelli RM, Ferraz MB, Santos WS, Meinão I, Quaresma MR. Tradução para a língua portuguesa e validação do questionário genérico de avaliação de qualidade de vida SF-36 (Brasil SF-36). Rev Bras Reumatol 1999; 39(3):143-150.
28. Malta DC, Cezário AC, Moura L, Morais Neto OL, Brito Junior J. A construção da vigilância e prevenção das doenças crônicas não transmissíveis no contexto do Sistema Único de Saúde. Epidemiol. Serv. Saúde 2006; 15(3):47-65.

29. Marcopito LF, Rodrigues SSF, Pacheco MA, Shirassu MM, Goldfeder AJ, Moraes MA. Prevalência de alguns fatores de risco para doenças crônicas na cidade de São Paulo. Rev Saude Publica 2005; 39(5):738-745.

30. Castro RAA, Moncau JEC, Marcopito LF. Prevalência de Hipertensão Arterial Sistêmica na Cidade de Formiga, MG. Arq Bras Cardiol 2007; 88(3):334-339.

31. Fuchs F, Moreira LB, Moraes RS, Bredemeir M, Cardozo SC. Prevalência de hipertensão arterial sistêmica e fatores associados na região urbana de Porto Alegre: estudo de base populacional. Arq Bras Cardiol 1995; 63(6):473-479.

32. Nascimento Neto RM, Krieger JE, Machado-Coelho GL. Projeto Corações do Brasil. Arq Bras Cardiol 2005; 85(3):218-221.

33. Barreto SM. Hypertension and clustering of cardiovascular risk factors in a community in Southeast Brazil - The Bambuí Health and Ageing Study. Arq Bras Cardiol 2001; 77(6):576-581.

34. Instituto Nacional de Câncer (Inca). Inquérito domiciliar sobre comportamentos de risco e morbidade referida de doenças e agravos não transmissiveis: Brasil, 15 capitais e Distrito Federal, 2002-2003. Rio de Janeiro: Inca; 2004.

35. Strine TW, Hootman JM, Chapman DP, Okoro CA, Balluz L. Healthrelated quality of life, health risk behaviors, and disability among adults with painrelated activity difficulty. Healthrelated quality of life, health risk behaviors, and disability among adults with pain-related activity difficulty. Am J Public Health 2005; 95(11):2042-2048.

36. Strine TW, Balluz L, Chapman DP, Moriarty DG, Owens M, Mokdad AH. Risk behaviors and healthcare coverage among adults by frequent mental distress status, 2001. Am J Prev Med 2004; 26(3):213-216.

37. Zack MM, Moriarty DG, Stroup DF, Ford ES, Mokdad AH. Worsening trends in adult health-related quality of life and self-rated health-United States, 1993-2001. Public Health Rep 2004; 119(5):493-505.

38. Ahluwalia IB, Holtzman D, Mack KA, Mokdad A. Health-related quality of life among women of reproductive age: Behavioral Risk Factor Surveillance System (BRFSS), 1998-2001. J Womens Health (Larchmt) 2003; 12(1):5-9.

39. Ahluwalia IB,Mack KA, Mokdad A, Indu B. Mental and Physical Distress Among Women. Obstetrics \& Gynecology 2004; 104(3):477

40. Nogales AMV. A mortalidade da população idosa no Brasil. Como vai? População brasileira. Brasília: IPEA; 1998

41. Bowling A, Gabriel Z, Dakes J, Dowding L, Evans $\mathrm{O}$, Fleissig A. Let's ask them: a national survey of definitions of quality of life and its enhancement among people aged 65 and over. Int J Aging Hum Dev 2003; 56(4):269-306.

42. Zunzunegui MV, Nunez O, Durban M, Garcia de Yébones MJ, Otero A. Decreasing prevalence of disability in activities of daily living, functional limitations and poor self-rated health: a 6 year follow-up study in Spain. Age Clin Exp Res 2006; 18(5):349-351. 
43. Felce D. Defining and applying the concept of quality of life. J Intellect Disabil Res 1997; 41(2):126-135.

44. Brazier JE, Harper R, Jones NMB, O'cathian A, Thomas KJ, Unsherwoodt WL. Validating the SF-36 health survey questionnaire: new outcome measure for primary care. BMJ 1992; 305(6846):160-164.

45. Perneger TV, Leplége A, Etter JF, Rougemont A. Validation of a French-language version of the MOS 36-Item Short-Form Health Survey (SF-36) in young healthy adults. J Clin Epidemiol 1995; 48(8):1051-1060.

46. Bullinger M. German translation and psychometric testing of the SF-36 health survey: preliminary results from the IQOLA project. Soc Sci Med 1995; 41(10):1359-1366.

47. Currey SS, Rao JK, Winfield JB, Callahan LF. Performance of a generic health-related quality of life measure in a clinic population with rheumatic disease. Arthritis Rheum 2003; 49(5):658-664.

48. Sullivan PW, Ghuschyan V, Wyatt HR, Wu EQ, Hill JO. Impact of Cardiometabolic Risk Factor Clusters on Health-related Quality of Life in the Unity States of America. Obesity 2007; 15(2):511-521.

49. Lee LM, Hsich S, Paffenbarger RS. Exercise intensity and longevity in men. The Harvard Alumni Healthy Study. JAMA 1995; 273(15):1179-1184.

50. Blair SN, Goodyear NN, Gibsons LW. Physical fitness and incidence of hypertension in healthy normotensive men. JAMA 1984; 252(4):487-490.

51. Cutler JA, Sorlie PD, Wolz M, Thom T, Fields LE, Roccella EJ. Trends in hypertension prevalence, awareness, treatment and control rates in United States adults between 1988-1994 and 1999-2004. Hypertension 2008; 52(5):818-827.

52. Erickson SR, Williams BC, Gruppen LD. Perceived symptoms and health-related quality of life reported by uncomplicated hypertensive patients compared to normal controls. J Hum Hypertens 2001; 15(8):539-548.

53. Wiklund I, Halling K, Ryden-Bergsten T, Fletcher A. Does lowering the blood pressure improve the mood? Quality-of-life results from the Hypertension Optimal Treatment (HOT) study. Blood Press 1997; 6(6):357-364.

54. Kumanyika SK, Obarzanek E, Stettler N, Bell R, Field AE, Fortmann SP, Franklin BA, Gillman MW, Lewis CE, Poston WC $2^{\text {nd }}$, Stevens J, Hong Y; American Heart Association Council on Epidemiology and Prevention, Interdisciplinary Committee for Prevention. Promotion of Healthful Eating, Physical Activity, and Energy Balance: A Population-Based Prevention of Obesity. The Need for Comprehensive Scientific Statement From American Heart Association Council on Epidemiology and Prevention, Interdisciplinary Committee for PreventionFormerly the Expert Panel on Population and Prevention Science) and Prevention. Circulation $2008 ; 118(4): 428-464$.

55. Silva MP Da, Jorge ZA, Domingues Nobre EL, Chambel P, De Castro JJ. Obesidade e qualidade de vida. Acta Med Port 2006; 19:247-250.

56. Ford SF, Moriarty DG, Zack MM, Mokdad DPC. Self-Reported Body Mass Index and Health-Related Quality of Life: Findings from the Behavioral Risk Factor Surveillance System. Obesity Research 2001; 9(1):21-31.
57. Silva MP Da, Jorge ZA, Domingues NEL. Chambel P, De Castro JJ. Obesidade e qualidade de vida. Acta Med Port 2006; 19:247-250.

58. Instituto Brasileiro de Geografia e Estatística (IBGE). Pesquisa de Orçamentos Familiares, 2002-2003. Rio de Janeiro: IBGE; 2006. (CD-ROM $3^{\text {a }}$ versão).

59. Wilson D, Parsons J, Wakefield M. The health-related quality- of life of never-smokers, ex-smokers, and light, moderate and heavy smokers. Prev Med 1999; 29(3):139-144.

60. Schumitz N, Kruse J, Kugker J. Disabilities, quality of life, and mental disorders associated with smoking and nicotine dependence. Am J Psychiatry 2003; 160(9):1670-1676.

61. Woolf SH, Rotemich SF, Johnson RE, Marsland DW. Is cigarette smoking associated with impaired physical and mental functional status? An office-based survey of primary care patients. Am J Prev Med 1999; 17(2):134-137.

62. Lyons RA, Lo SV, Littlepage BNC. Perception of health amongst ever-smokers and never-smokers: a comparison using the SF-36 Health Survey Questionnaire. Tob Control 1994; 3(3):213-215.

63. Mitra M, Chung M, Wilber N, Walker D. Smoking status and quality of life. A longitudinal study among adults with disabilities. Am J Prev Med 2004; 27(3):258-260.

64. Breslau N, Novak S, Kessler R. Psychiatric disorders and stages of smoking. Biol Psychiatry 2004; 55(1):69-76.

65. Cayela A, Rodrigues- Dominguez S, Otero R. Deteriorated Health-Related Quality of Life in Healthy Male Smokers. Archivos de Bronconeumologia 2007; 43(2):59-63.

66. Tilmann M, Silcok JA. Comparison of smokers' and ex-smokers' healthrelated quality of life. $J \mathrm{Pu}$ blic Health Med 1997; 19(3):268-273.

67. Olufade A, Shaw JW, Foster S, Leischow S, Hays R, Coons SJ. Development of the Smoking Cessation Quality of Life Questionnaire. Clin Ther 1999; 21(12):2113-2130.

68. Volk RJ, Cantor SB, Steinbauer JR, Cass AR. Alcohol use disorders, consumption patterns, and health-related quality of life of primary care patients. Alcohol Clin Exp Res 1997; 21(5):899-905.

69. Kraemer KL, Stephen AM, Conigliaro J, McNeil M, Gordon AJ, Kelley ME. Decreased Alcohol Consumption in Outpatient Drinkers Is Associated with Improved Quality of Life and Fewer Alcohol-related Consequences. J Gen Intern Med 2002; 17(5):382-386.

70. Rhem J, Gmel G, Sempos CT, Trevisan M. Alcohol-related morbidity and mortality. Alcohol Res Health 2003; 27(1):39-51.

71. Kraemer KL, Stephen AM, Conigliaro J, McNeil M, Gordon AJ, Kelley ME. Decreased Alcohol Consumption in Outpatient Drinkers Is Associated with Improved Quality of Life and Fewer Alcohol-related Consequences. J Gen Intern Med 2002; 175(5): 382-386.

Artigo apresentado em 25/11/2011

Aprovado em 20/02/2012

Versão final apresentada em 28/02/2012 